\title{
Effect of Pressure on Strongly Polar Liquid Crystals
}

\author{
S. Krishna Prasad*, S. Pfeiffer**, G. Heppke**, and R. Shashidhar*
}

* Raman Research Institute, Bangalore 560080, India

** Technische Universität Berlin, ER 11, Straße des 17. Juni 135, D-1000 Berlin 12, Germany

Z. Naturforsch. 40 a, 632-635 (1985); received March 6, 1985

The effect of pressure on several mesogens which possess a strongly polar terminal group (CN or $\left.\mathrm{NO}_{2}\right)$ is studied. In all cases the partially bilayer smectic $\mathrm{A}\left(\mathrm{A}_{d}\right)$ gets bounded while the nematic phase gets more stabilised at high pressures. In the case of reentrant nematogens, the maximum pressure of smectic stability is seen to be related to the temperature range of the nematic phase at 1 bar.

\section{Introduction}

Reentrant nematic behaviour is known to be exhibited by two phenyl as well as three phenyl ring systems with a strongly polar cyano group attached to one end of the molecule [1]. A few compounds with a nitro end group have also shown reentrant polymorphism, but such instances are relatively few. High pressure studies on 8 single component systems which exhibit the reentrant nematic $\left(\mathrm{N}_{\mathrm{re}}\right)$ phase at atmospheric pressure were reported in an earlier paper [2]. In this paper we present results of our high pressure studies on several materials, binary mixtures as well as pure compounds, whose molecular structure is known to be favourable to the occurrence of reentrant nematic behaviour.

\section{Materials}

The substances studied are listed below:

1) Binary mixtures of 4-n-octyloxy-4'-n-cyanobiphenyl (8 OCB)

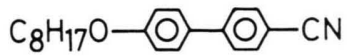

and 4-cyano benzylidene-4'-n-octyloxyaniline (CBOOA)

$$
\mathrm{C}_{8} \mathrm{H}_{17} \mathrm{O}-\mathrm{O}-\mathrm{N}=\mathrm{CH}-\mathrm{C}-\mathrm{CN}
$$

2) 4-cyanophenyl-4'-(4"'-nonyloxy benzoyloxy)benzoate (9OCPBB)

$$
\mathrm{C}_{9} \mathrm{H}_{19} \mathrm{O}-\mathrm{O}-\mathrm{COO}-\mathrm{O}-\mathrm{COO}-\mathrm{O}-\mathrm{CN}
$$

Reprint requests to Prof.Dr. G. Heppke, Technische Universität Berlin, ER11, Straße des 17. Juni 135, D-1000 Berlin 12.
3) 4-cyano benzylidene-4'-n-nonyloxyaniline (CBNA)

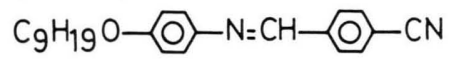

4) 4-cyanophenyl-4'-n-nonyloxy benzoate (CPNB)

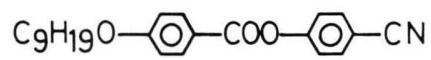

5) 4-nitrobenzylidene-4'-n-octyloxyaniline (NBOOA)

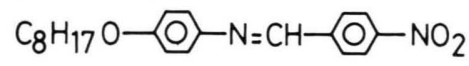

The transition temperatures of these substances are listed in Table 1.

The smectic A phases of $\mathrm{CBOOA}$ and $8 \mathrm{OCB}$ have been studied by X-ray diffraction [3,4]. For both the smectic A phase has been characterised as a partially bilayer $A$ phase $\left(A_{d}\right)$ with a $d / l$ ratio of about 1.4 , which is practically independent of tem-

Table 1. Transition temperatures in ${ }^{\circ} \mathrm{C}$ (at 1 bar) of the

\begin{tabular}{|c|c|c|c|c|c|}
\hline Substance & $\mathrm{K}$ & $\mathrm{N}_{\mathrm{re}}$ & $\mathrm{S}_{\mathrm{A}}$ & $\mathrm{N}$ & I \\
\hline \multicolumn{6}{|c|}{ 8OCB/CBOOA mixtures } \\
\hline$X=0$ & +73.0 & - & +82.5 & +107.2 & + \\
\hline$X=0.1$ & +67.5 & - & +81.5 & +104.4 & + \\
\hline$X=0.3$ & +59.0 & - & +78.0 & +98.4 & + \\
\hline$X=0.5$ & +39.0 & - & +75.1 & +91.9 & + \\
\hline$X=0.7$ & +47.0 & - & +71.2 & +87.3 & + \\
\hline$X=0.9$ & +53.0 & - & +67.5 & +82.0 & + \\
\hline$X=1.0$ & +55.0 & - & +66.8 & +79.9 & + \\
\hline 9 OCPBB & +120.4 & $+(116.4)$ & +194.8 & +226.4 & + \\
\hline CPNB & +76.7 & - & $+(58.6)$ & +81.5 & + \\
\hline CBNA & +72.0 & - & +97.5 & +103.6 & + \\
\hline NBOOA & +62.5 & - & +74.0 & +84.2 & + \\
\hline
\end{tabular}
compounds studied.

( ) denotes that the transition is monotropic; K, crystal; $\mathrm{N}_{\text {re }}$, reentrant nematic; $\mathrm{A}$, smectic $\mathrm{A} ; \mathrm{N}$, nematic; I, isotropic. 
Table 2. $d$ and $d / l$ values for the substances used in the pressure study.

\begin{tabular}{lll}
\hline Compound & $d(\AA)$ & $d / l$ \\
\hline 9OCPBB & 37.9 & 1.15 \\
CPNB & 33.8 & 1.26 \\
CBNA & 36.5 & 1.35 \\
NBOOA & 31.6 & 1.25 \\
\hline
\end{tabular}

perature ( $d$ is the layer thickness in the A phase as measured by X-ray diffraction, while $l$ corresponds to the length of the molecule in its most extended configuration as obtained by a molecular model). We have determined $d$ for compounds $2-5$ at a common relative temperature of $\left(T_{\mathrm{AN}}-5\right){ }^{\circ} \mathrm{C}$. These values of $d$ along with the $d / l$ values are listed in Table 2. It is clear that the A phase of these compounds can be categorised as an $A_{d}$ phase.

\section{Experimental}

An optical high pressure cell [2] was used for the experiment. The liquid crystalline sample was sandwiched between two optically polished sapphire cylinders which are enclosed in an elastomer tube (Fluran). This ensured that the sample is isolated from the pressure transmitting fluid (plexol). Transition temperatures were determined by varying the temperature at a constant pressure and monitoring the changes in the intensity of the laser light transmitted by the sample. Pressures were measured by a Heise gauge to an accuracy of \pm 1 bar while transition temperatures were measured to $\pm 0.1^{\circ} \mathrm{C}$.

\section{Results and Discussion}

\section{1. $8 O C B / C B O O A$ mixture}

We have studied the effect of pressure on the smectic A-nematic $(A-N)$ phase boundary in the $\mathrm{P}-\mathrm{T}$ diagram for 5 different $8 \mathrm{OCB} / \mathrm{CBOOA}$ mixtures, viz. $X=0.1,0.3,0.5,0.7,0.9$ (where $X$ is the mole fraction of $9 \mathrm{OCB}$ in the mixture). We have also studied, for the sake of completeness, the A-N boundary of both pure $8 \mathrm{OCB}$ and $\mathrm{CBOOA}$ at high pressure. The $\mathrm{P}-\mathrm{T}$ diagram for the mixtures as well as the pure compounds is presented in Figure 1. It is seen that in each case the $\mathrm{A}-\mathrm{N}$ phase boundary has the characteristic elliptic shape [2] and also there exists a maximum pressure of smectic stability $\left(P_{\mathrm{m}}\right)$ beyond which the A phase ceases to exist.

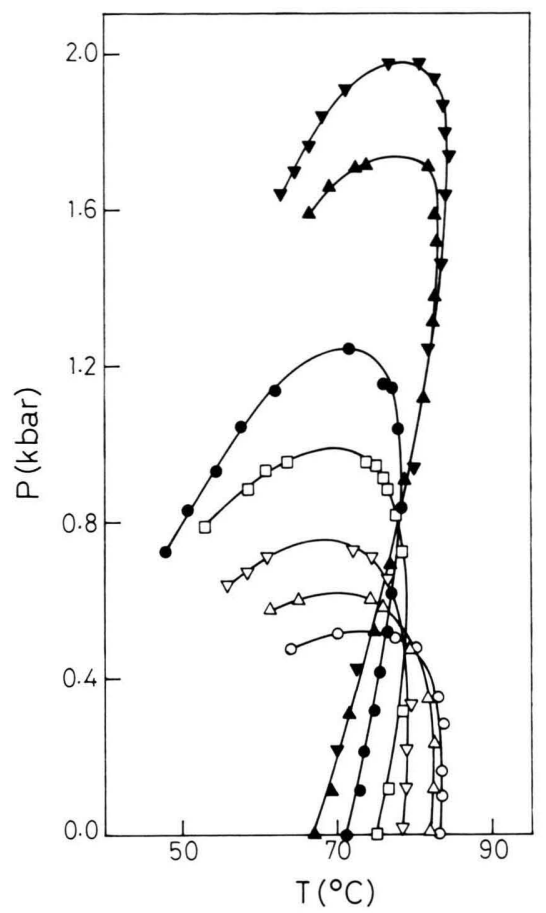

Fig. 1. $\mathrm{P}-\mathrm{T}$ diagram of $8 \mathrm{OCB}(\boldsymbol{\nabla}), \mathrm{CBOOA}(\mathrm{O})$ and $8 \mathrm{OCB} / \mathrm{CBOOA}$ mixtures, showing the smectic A-nematic phase boundary. The concentrations of the mixtures are $X=0.9(\Delta), X=0.7(\bullet), X=0.5(\square), X=0.3(\nabla)$ and $X=0.1(\triangle)$, where $X$ is the mole fraction of $8 \mathrm{OCB}$ in the mixture.

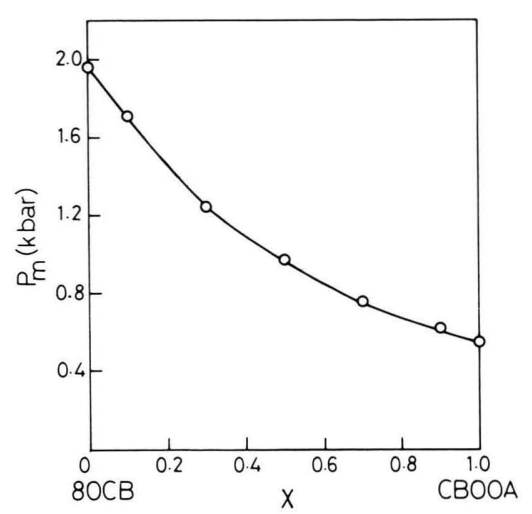

Fig. 2. Variation of the maximum pressure of smectic stability $\left(P_{\mathrm{m}}\right)$ with concentration for $8 \mathrm{OCB} / \mathrm{CBOOA}$ mixtures.

The variation of $P_{\mathrm{m}}$ with $X$ is, as to be expected from data for other binary mixtures [5-7], a smooth curve (Figure 2). It might be recalled that Herrmann [8] has also measured $P_{\mathrm{m}}$ for several mixtures of $8 \mathrm{OCB} / \mathrm{CBOOA}$. He found that $P_{\mathrm{m}}$ shows a nonlinear variation with $X$, there being a maximum 


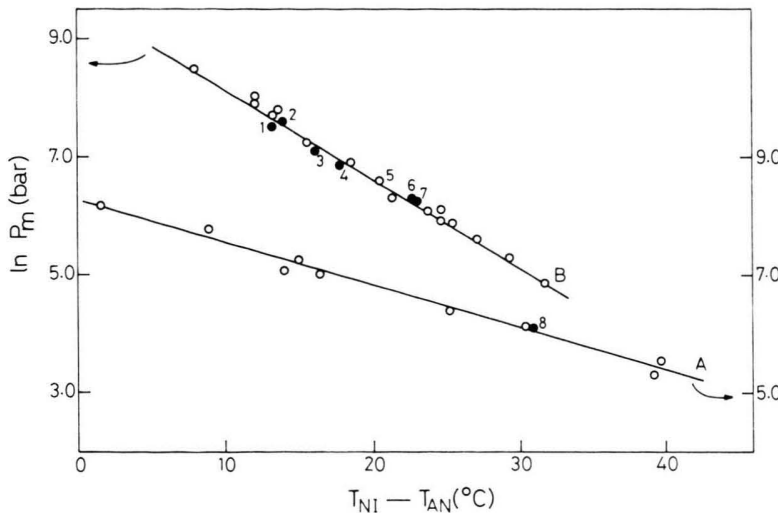

Fig. 3. Dependence of $P_{\mathrm{m}}$ on the range of the nematic phase $\left(T_{\mathrm{NI}}-T_{\mathrm{AN}}\right)$ at atmospheric pressure. The open circles are data from [2], while the closed circles are data discussed in this paper. The points 1 and 7 correspond to $8 \mathrm{OCB}$ and $\mathrm{CBOOA}$, respectively, while the points $2-6$ are for $8 \mathrm{OCB} / \mathrm{CBOOA}$ mixtures with concentrations $X=0.9$ (2), 0.7 (3), 0.5 (4), 0.3 (5), 0.1 (6), respectively. The point 8 is for $9 \mathrm{OCPBB}$, a three phenyl ring compound (see text).

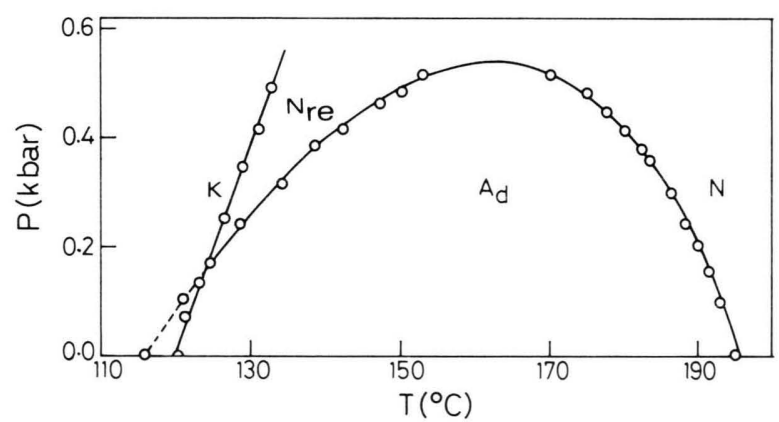

Fig. 4. $\mathrm{P}-\mathrm{T}$ diagram of $9 \mathrm{OCPBB}$ showing the $\mathrm{A}-\mathrm{N}$ phase boundary.

for $X \simeq 0.3$. Our results are in disagreement with those of Herrmann. In an earlier paper [2] we had shown that $P_{\mathrm{m}}$ is uniquely related to the range of the nematic phase, $R\left(=T_{\mathrm{NI}}-T_{\mathrm{AN}}\right)$, at 1 bar by the relation $P_{\mathrm{m}}=P_{0} \exp (-m R)$ where $P_{0}$ and $m$ are constants which depend only on the number of phenyl rings in the molecule. To check whether our present results on $8 \mathrm{OCB} / \mathrm{CBOOA}$ also fit this relation we have plotted in Fig. 3 our data for the $8 \mathrm{OCB} / \mathrm{CBOOA}$ mixtures along with all the earlier data given by Kalkura et al. [2]. It is seen that the $8 \mathrm{OCB} / \mathrm{CBOOA}$ data indeed fit very well the line $\mathrm{B}$, which is the line for two phenyl ring systems. The discrepancy of our data compared to those of [8] is perhaps attributable to the thermal degradation of CBOOA in Herrmann's measurements, conceivably caused by the sample being in direct contact with the steel gasket.

\section{2. $9 O C P B B$}

The $\mathrm{P}-\mathrm{T}$ diagram of $9 \mathrm{OCPBB}$, a three phenyl ring system, which exhibits reentrant nematic at atmospheric pressure, is given in Figure 4. It is seen that the $\mathrm{A}-\mathrm{N}$ phase boundary again has the elliptic shape. The value of $P_{\mathrm{m}}=540 \mathrm{bar}$ fits the line A of Fig. 3 showing once more the validity of the relation discussed earlier.

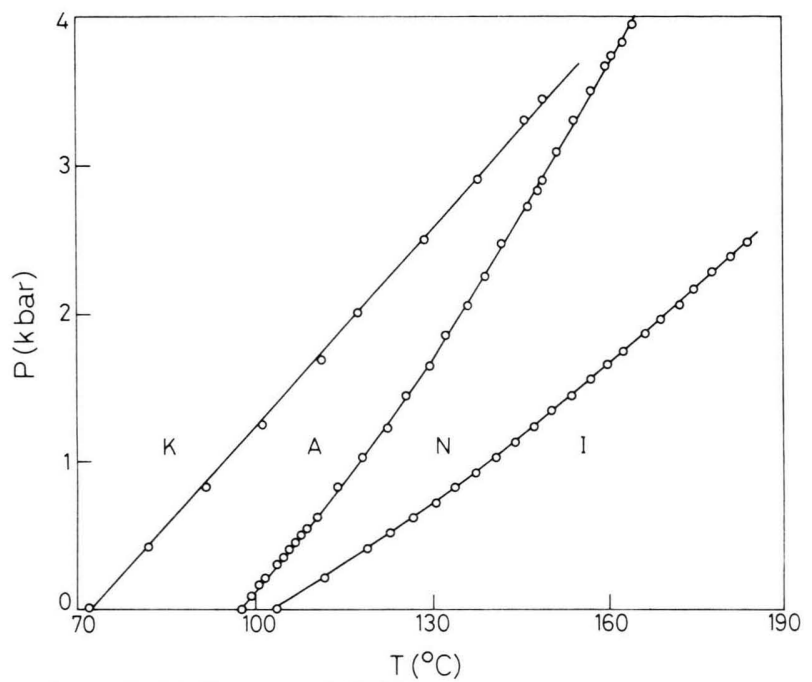

Fig. 5. $\mathrm{P}-\mathrm{T}$ diagram of $\mathrm{CBNA}$.

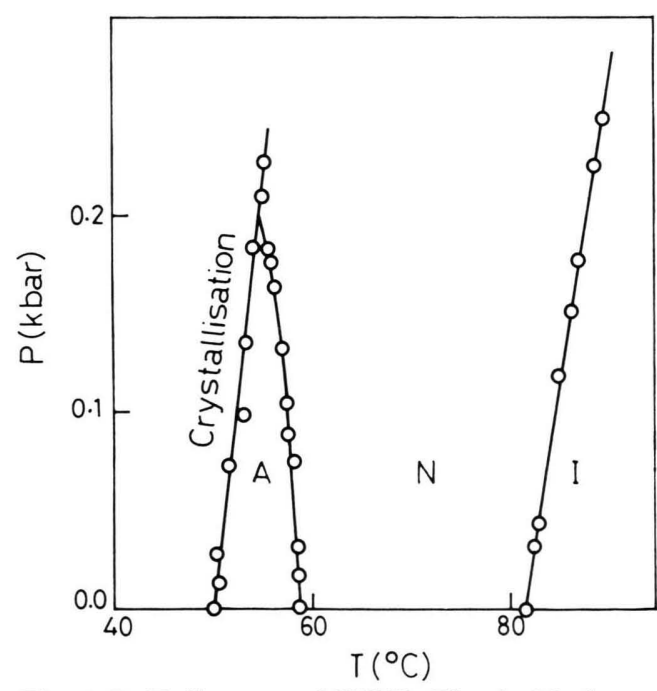

Fig. 6. $\mathrm{P}-\mathrm{T}$ diagram of $\mathrm{CPNB}$. The $\mathrm{A}-\mathrm{N}$ phase transition is monotropic at all pressures. 


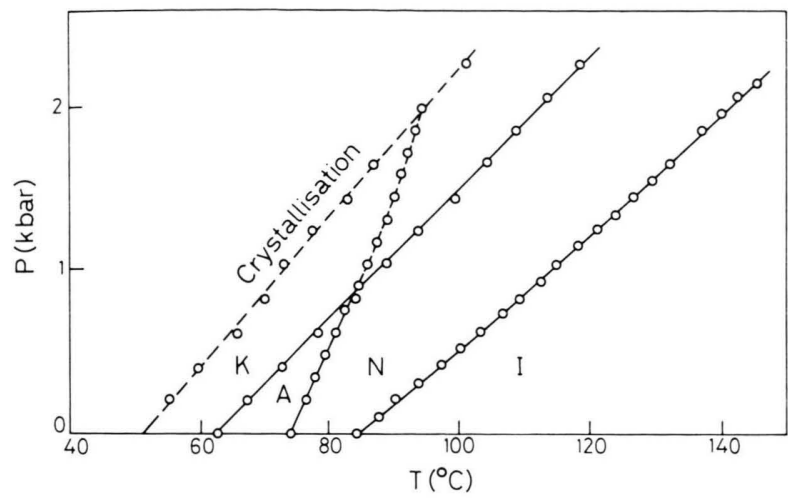

Fig. 7. P-T diagram of NBOOA. The dashed lines indicate that the transitions are monotropic.

\section{3. $C B N A$}

This compound is the next higher homologue of CBOOA. Considering that CBOOA exhibits the reentrant nematic phase at a relatively low pressure (see Fig. 1), it was expected that CBNA would also show an RN phase. But the $\mathrm{P}-\mathrm{T}$ diagram (Fig. 5) shows that the $A_{d}$ phase of CBNA is surprisingly stable over a wide range of pressures. There is of course a decrease of the $A_{d}$ range with increase of pressure but the rate of this decrease is quite small, and also the $\mathrm{A}-\mathrm{N}$ phase boundary does not show a pronounced curvature towards the pressure axis even up to $4 \mathrm{kbar}$, the highest pressure that we have reached. Extrapolating the melting and $\mathrm{A}-\mathrm{N}$ boundaries to higher pressures, we expect the $A_{d}$ phase to be bounded (in the heating mode) at $\approx 4.3 \mathrm{kbar}$.

Addition of an extra methylene group to the end chain (compared to CBOOA) therefore seems to enhance considerably the stability of the $A_{d}$ phase.

\section{4. $C P N B$}

This is a two phenyl ring compound with a $\mathrm{COO}$ bridging group. The phase diagram of this com-

[1] Nguyen Huu Tinh, J. Chim. Phys. 80, 83 (1983).

[2] A. N. Kalkura, R. Shashidhar, and M. Subramanya Raj Urs, J. Physique. 44, 51 (1983).

[3] A. J. Leadbetter, J. C. Frost, J. P. Gaughan, G. W. Gray, and A. Mosley, J. Physique 40, 375 (1979).

[4] D. Guillon, P. Seurin, and A. Skoulios, Mol. Cryst. Liq. Cryst. 51, 149 (1979).

[5] P. E. Cladis, R. K. Bogardus, W. B. Daniels, and G. N. Taylor, Phys. Rev. Lett. 39, 720 (1977).

[6] P. E. Cladis, in 'Liquid Crystals' - Proc. Int. Liq. Cryst. Conf., Bangalore (Ed.: S. Chandrasekhar), Heyden, London 1980, p. 105. pound is given in Figure 6. It is seen that the A-N has a negative slope throughout and $\mathrm{A}_{d}$ gets bounded at 200 bars. It is obvious that this compound would surely have exhibited the $\mathrm{N}_{\mathrm{re}}$ phase at a higher pressure if crystallisation had not intervened.

\subsection{NBOOA}

It is relevant to recall that so far [9] only one three phenyl ring nitro compound in which the longitudinal components of the bridging dipoles are additive with respect to the dipole of the nitro end group has shown a reentrant nematic phase at high pressure. We undertook high pressure studies in order to see if such a possibility exists in this terminally nitro substituted compound which has only two phenyl rings. Figure 7 gives the $\mathrm{P}-\mathrm{T}$ diagram for NBOOA. It can be seen that the $\mathrm{A}-\mathrm{N}$ transition, which is enantiotropic at lower pressures, becomes monotropic beyond $1.8 \mathrm{kbar}$. Finally, at $\approx 2 \mathrm{kbar}$, the crystallisation line cuts the $\mathrm{A}-\mathrm{N}$ line and thereby the A phase ceases to exist for pressures beyond $2 \mathrm{kbar}$. Also the $\mathrm{A}-\mathrm{N}$ line is practically linear throughout, and absence of any curvature of the boundary precludes the possibility of observing the $\mathrm{N}_{\text {re }}$ phase.

These studies show that although pressure does have the effect of destabilizing the $A_{d}$ phase in terminally polar liquid crystals, the occurence of reentrant nematic is clearly less favoured in two phenyl ring systems than in three phenyl ring systems.

\section{Acknowledgements}

We are thankful to Prof. S. Chandrasekhar and Dr. B. R. Ratna for discussions. One of us (SP) acknowledges the financial support of the Gesellschaft von Freunden der Technischen Universität Berlin.

[7] R. Shashidhar, H. D. Kleinhans, and G. M. Schneider, Mol. Cryst. Liq. Cryst. 72 (Lett.), 119 (1981).

[8] J. Herrmann, Mol. Cryst. Liq. Cryst. 72 (Lett.), 219 (1982).

[9] B. R. Ratna, S. Krishna Prasad, R. Shashidhar, G. Heppke, and S. Pfeiffer, Presented at the X. International Liquid Cryst. Conf., York, July 1984, Mol. Cryst. Liq. Cryst. (in press). 